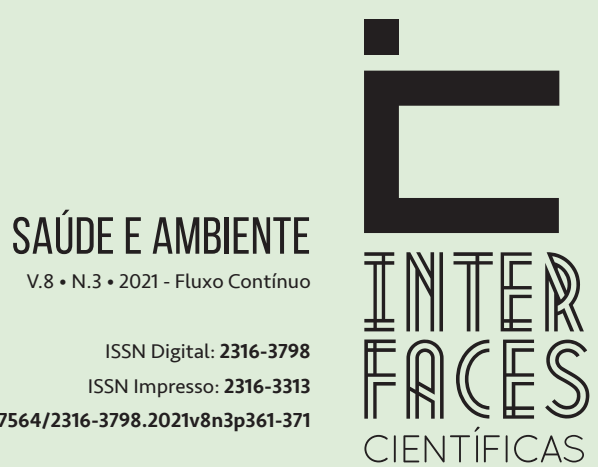

\section{AVALIAÇÃO DA NATREMIA EM PORTADORES DA IMUNODEFICIÊNCIA ADQUIRIDA}

SERUM SODIUM LEVEL EVALUATION IN ACQUIRED IMMUNODEFICIENCY PATIENTS

\section{EVALUACIÓN DE NATREMIA EN PACIENTES CON INMUNODEFICIENCIA ADOUIRIDA}

Isabelle Araujo de Oliveira Santana Daniel Silva Santos ${ }^{2}$

Francisco de Assis Pereira ${ }^{3}$

Luan Fellipe Bispo Almeida ${ }^{4}$

\section{RESUMO}

O objetivo deste estudo foi avaliar o nível sérico de sódio, no momento da admissão, de pacientes hospitalizados com diagnóstico de Vírus da Imunodeficiência Humana / Síndrome da Imunodeficiência Adquirida (HIV/SIDA), assim como descrever características clínicas e achados laboratoriais dos vírus. Tratou-se de um estudo descritivo, observacional e transversal com abordagem quantitativa e amostragem de conveniência de 19 pacientes com diagnóstico de HIV/SIDA hospitalizados na Enfermaria de Doenças Infecciosas do Hospital Universitário da Universidade Federal de Sergipe (HU-UFS). Para a coleta de dados e a consecução de objetivos do estudo, foi realizada revisão dos prontuários médicos dos pacientes internados no Hospital Universitário, comparando os resultados com um grupo controle (GC) de indivíduos saudáveis pareados por idade, sexo e altura. Entre os pacientes HIV/SIDA avaliados, 57,9\% apresentaram hiponatremia e houve diferença estatística quando comparados os GC e o grupo HIV/SIDA (GS) (GC $=140,2 \pm 1,4$ vs GS $=133,1 \pm 5,6, p<0,001)$. O GS também apresentou maior frequência de anemia, hipoalbuminemia, elevação de gama-GT; além de valores estatitisticamente menores de peso e IMC. A hiponatremia é uma situação clínica frequente em pacientes com HIV/SIDA, a qual deve ser diagnosticada e tratada precoce e adequadamente.

\section{PALAVRAS-CHAVE}

Vírus da Imunodeficiência Humana. Hiponatremia. Síndrome da Imunodeficiência Adquirida. 


\section{ABSTRACT}

The objective of this study was to evaluate the serum sodium level at the time of admission of hospitalized patients diagnosed with Human Immunodeficiency Virus / Acquired Immune Deficiency Syndrome (HIV / AIDS), as well as to describe clinical characteristics and laboratory findings. It was a descriptive, observational and cross-sectional study with quantitative approach and convenience sample of 19 patients diagnosed with HIV / AIDS hospitalized in the Infectious Diseases Ward of the University Hospital of the Federal University of Sergipe (HU-UFS). For the collection of data and the achievement of study objectives, a review of the medical records of hospitalized patients at the University Hospital was carried out, comparing the results with a control group (CG) of healthy individuals matched by age, sex and height. Among the HIV / AIDS patients evaluated, $57.9 \%$ presented hyponatremia and there was statistical difference when compared to the CG and the HIV / AIDS group (GS) (GC = 140.2 \pm 1.4 VS GS $=133.1 \pm 5$, $6, p<0.001$ ). GS also presented higher frequency of anemia, hypoalbuminemia, elevation of gamma-GT; In addition to statistically lower values of weight and BMI. Hyponatremia is a frequent clinical condition in patients with HIV / AIDS, which should be diagnosed and treated early and adequately.

\section{KEYWORDS}

Human Immunodeficiency Virus; Hyponatremia; Acquired Immune Deficiency Syndrome.

\section{RESUMEN}

El objetivo de este estudio fue evaluar el nivel de sodio sérico, en el momento del ingreso, de pacientes hospitalizados con diagnóstico de Virus de Inmunodeficiencia Humana/Síndrome de Inmunodeficiencia Adquirida (VIH/ SIDA), así como describir sus características clínicas y hallazgos de laboratorio. Fue un estudio descriptivo, observacional y transversal con abordaje cuantitativo y muestreo por conveniencia de 19 pacientes diagnosticados de VIH/SIDA hospitalizados en la Sala de Enfermedades Infecciosas del Hospital Universitario de la Universidad Federal de Sergipe (HU-UFS). Para recolectar datos y alcanzarlos objetivos del estudio, se realizó una revisión de la historia clínica de los pacientes ingresados en el Hospital Universitario, comparando los resultados con un grupo control (GC) de individuos sanos emparejados por edad, sexo y altura. Entre los pacientes con VIH / SIDA evaluados, el $57,9 \%$ presentaba hiponatremia y hubo diferencia estadística al comparar el grupo GC y el grupo VIH / SIDA (GS) (GC = 140,2 $\pm 1,4$ y GS = 133,1 \pm 5,6; $p<0,001)$. El GS también mostró una mayor frecuencia de anemia, hipoalbuminemia, elevación de gamma-GT; además de valores estadísticamente más bajos de peso e IMC. La hiponatremia es una situación clínica común en pacientes con VIH/SIDA, que debe ser diagnosticada y tratada de manera precoz y adecuada. 


\section{PALABRAS CLAVE}

Virus de la inmunodeficiencia humana; Hiponatremia; Síndrome de inmunodeficiencia adquirida.

\section{INTRODUCÇÃO}

A infecção pelo vírus da imunodeficiência humana/síndrome da imunodeficiência adquirida (HIV/ SIDA) é um importante problema de saúde pública. Desde a sua descoberta, em 1981 nos Estados Unidos, houve um incremento substancial no número de casos notificados (MELO, 2020). Apesar da elevada incidência, tem se observado uma diminuição do número de óbitos e aumento da sobrevida desses pacientes, o que reflete, sobretudo, o impacto positivo da terapia antirretroviral (TARV) (MALHEIROS et al., 2019).

Com o aumento da expectativa de vida, surgiram diversas complicações inerentes à infecção pelo HIV, bem como ao uso de medicações decorrentes desta. Entre elas, destaca-se a hiponatremia, distúrbio hidroeletrolítico mais comum em pacientes com HIV/SIDA (MENON et al., 2007). Madariaga e colaboradores (2015) realizaram uma pesquisa a fim de demonstrar a ocorrência de hiponatremia em pacientes hospitalizados com diagnóstico de HIV/SIDA e observaram que a prevalência de hiponatremia varia de 20 a $80 \%$ e essa porcentagem depende tanto dos critérios diagnósticos para sua definição quanto da população estudada.

A população com HIV/SIDA compartilha uma série de fatores de risco adicionais para o desenvolvimento da hiponatremia, pelos mais variados mecanismos. Entre eles, a síndrome da secreção inapropriada do hormônio antidiurético (SIADH) (deflagrada por infecções oportunistas, como tuberculose, toxoplasmose e pneumocistose), síndrome cerebral perdedora de sal (induzida pelo uso de medicações como lopinavir/ritonavir e sulfametaxazol/trimetropin) e o desenvolvimento de resistência parcial à ação dos glicocorticoides (GINĖS; GUEVARA, 2008).

A hiponatremia neste grupo de pacientes além de ser bastante comum, está relacionada a pior prognóstico, maior tempo de hospitalização e maiores taxas de mortalidade quando compara a pacientes HIV eunatrêmicos (GUEVARA, 2008).

O presente estudo objetivou avaliar os níveis séricos de sódio em pacientes hospitalizados com diagnóstico de HIV/SIDA, bem como suas características clínicas e achados laboratoriais.

\section{MATERIAIS E MÉTODOS}

Tratou-se de um estudo descritivo, observacional e transversal com abordagem quantitativa e amostragem de conveniência. A coleta foi realizada na enfermaria de doenças infecciosas de um hospital público terciário de referência para o tratamento de pacientes portadores de HIV/SIDA, localizado em Aracaju/SE, no período de agosto a novembro de 2016. 
Foram incluídos todos os pacientes maiores de 18 anos, com diagnóstico confirmado de HIV/SIDA. Os critérios de exclusão foram: ter idade inferior a 18 anos, síndromes genéticas, transtornos alimentares, gestação, hipotireoidismo, insuficiência renal, insuficiência cardíaca, tabagismo e etilismo.

Este estudo não teve conflitos de interesse e foi aprovado pelo Comitê de Ética em Pesquisa sob CAAE: 64009416.8.0000.5546, parecer número 1921470.

\subsection{COLETA DE DADOS}

A amostra foi dividida em dois grupos, o grupo doença (GS), composto por 19 pacientes com diagnóstico confirmado de HIV/SIDA e o grupo controle (GC), formado por 19 indivíduos saudáveis. Ambos os grupos foram pareados por sexo, idade e altura.

As informações acerca dos pacientes foram coletadas e registradas por meio do preenchimento de ficha-protocolo contendo nome, sexo, idade, peso, altura, índice de massa corpórea (IMC), medicamentos em uso.

O tempo de diagnóstico de HIV/SIDA foi registrado a partir do diagnóstico laboratorial e o tempo de tratamento para HIV/SIDA a partir do início do uso de antirretro-virais. Dados laboratoriais foram avaliados mediante coleta de sangue venoso durante as primeiras 24 horas de hospitalização, após jejum de 10 a 12 horas, no laboratório de análises clínicas do hospital. Os valores de hormônio tireoestimulante (TSH) e tireotoxina livre $\left(\mathrm{T}_{4} \mathrm{~L}\right)$ foram determinados por quimioluminescência; Triglicerídeos, lipoproteínas de alta densidade (HDL), lipoproteínas de baixa densidade (LDL) e colesterol total (CT) pelo método enzimático em mg/dl; ureia e creatinina pelo método cinético; sódio e potássio por eletrodos de referência; albumina por calorimetria; transaminase glutâmica oxalacética (TGO) e transaminase glutâmico pirúvica (TGP) por UV otimizado; gama-GT por SZASZ modificado; e hemograma por citometria de fluxo a LASER. Os resultados de CD4+ e carga viral foram obtidos do prontuário dos pacientes.

Também, foi realizada uma análise comparativa entre os pacientes portadores de HIV/SIDA eunatrêmicos (GI) e hiponatrêmicos.(GII)

\subsection{ANÁLISE ESTATÍSTICA}

Para a análise estatística dos resultados obtidos, utilizou-se o programa GraphPad Prism, versão 7.0 para Windows, 2016 (San Diego, CA, USA). Os resultados foram apresentados como média e desvio-padrão. Para a análise das variáveis paramétricas foi utilizado o teste t de Student e para as não-paramétricas o teste de Mann Whitney. As correlações entre os diversos parâmetros estudados foram realizadas por meio do coeficiente de variação Pearson para variáveis paramétricas e Spearman para variáveis não-paramétricas. Para a análise comparativa das variáveis de contingência entre os grupos, utilizou-se o teste Qui-quadrado. 0 nível de significância adotado para rejeição da hipótese de nulidade foi de $5 \%(p<0,05)$. 


\section{RESULTADOS}

Em ambos os grupos houve uma predominância do sexo feminino 12 (63,2\%). A média de peso (Kg) para o GC foi de 68,5 $\pm 6,5$ e para o GS foi de 60,2 $\pm 12,5$, com $p$-valor $=0,01$. Em relação ao IMC $\left(\mathrm{kg} / \mathrm{m}^{2}\right)$, a média para o GC foi de25,2 $\pm 0,7$ e para o GS de $22,4 \pm 0,4(p=0,01)$.

Em relação ao tempo de diagnóstico e de tratamento com antirretrovirais foi de 52,8 $\pm 42,7$ para o GC e 50,7 44,4 meses para o GS. Quanto aos níveis de CD4+ e a carga viral dos indivíduos do GS foram de 291,1 $\pm 326,9$ (células/ $\mu$ l) e $442638 \pm 812780$ (cópias $/ \mathrm{ml}$ ), respectivamente. Dos 19 pacientes, seis $(31,5 \%)$ tinham carga viral indetectável, dos quais três eram eunatrêmicos e três hiponarêmicos. A partir da correlação entre CD4+ e os níveis séricos de sódio, foi observado $r=0,42 ; p=0,07$ e na correlação entre carga viral e níveis séricos de sódio foi obtido $r=-0,65 ; p=0,001$.

No tocante ao esquema medicamentoso utilizado pelos pacientes do GS, verificou-se que nove $(47,4 \%)$ estavam em uso de Tenofovir + Lamivudina + Efavirenz, três (15,8\%) de Zidovudina + Lamivudina + Lopinavir/Ritonavir, dois (10,5\%) de Tenofovir + Lamivudina + Atazanavir/Ritonatir, dois (10,5\%) de Tenofovir + Lamivudina + Lopinavir/Ritonavir; um (5,3\%) de Didanosina + Lamivudina + Atazanavir/ Ritonavir, um (5,3\%) de Didanosina + Lamivudina + Raltegravir e um (5,3\%) de Lamivudina + Abacavir + Atazanavir/Ritonavir. Da casuística, somente um paciente fazia uso do Sulfametaxazol/Trimetropin.

Os resultados obtidos referentes aos parâmetros bioquímicos avaliados nos indivíduos do GC e do GS estão apresentados na Tabela 1. A hiponatremia esteve presente em 57,9\% dos pacientes com HIV/SIDA hospitalizados no período do estudo. Quando comparado com o GC, observou-se diferença estatística $(p<0,001)$. Nos grupos avaliados houve diferença estatística nos seguintes parâmetros bioquímicos: hemácias, hemoglobina, hematócrito, ureia, creatinina, albumina, TGO, gama-GT, TSH, $\mathrm{T}_{4} \mathrm{~L}$, HDL e LDL (Tabela 1).

Tabela 1 - Parâmetros bioquímicos avaliados nos indivíduos do grupo controle (GC) e nos pacientes portadores da síndrome da imunodeficiência adquirida (GS) internados na enfermaria de doenças infecciosas de um hospital público terciário de referência para o tratamento de pacientes portadores de HIV/SIDA, localizado em Aracaju - Sergipe, no período de agosto a novembro de 2016

\begin{tabular}{cccc}
\hline & GC $(\mathbf{n}=\mathbf{1 9})$ & $\mathbf{G S}(\mathbf{n}=\mathbf{1 9})$ & $\mathbf{p}$ \\
\hline HEMÁCIAS (milhões/mm3) & $4, \pm 0,5$ & $3,43 \pm 0,7$ & 0,001 \\
HEMOGLOBINA (g/dL) & $13,2 \pm 0,6$ & $10,0 \pm 2,6$ & 0,001 \\
HEMATÓCRITO (\%) & $40,3 \pm 1,9$ & $30,8 \pm 7,7$ & 0,001 \\
LEUCOCITOS (mm3) & $8393 \pm 2733$ & $8177,0 \pm 4638$ & 0,43 \\
PLAQUETAS (mm3) & $308.634 \pm 81.351$ & $273.074 \pm 103.561$ & 0,24 \\
UREIA (mg/dL) & $37,3 \pm 2,8$ & $30,8 \pm 12,5$ & 0,001 \\
CREATININA (mg/dL) & $1,11 \pm 0,2$ & $0,85 \pm 0,40$ & 0,001 \\
\hline
\end{tabular}




\begin{tabular}{cccc}
\hline & GC $(\mathbf{n}=\mathbf{1 9})$ & GS $(\mathbf{n}=\mathbf{1 9})$ & p \\
\hline POTÁSSIO (mmol/L) & $4,0 \pm 0,4$ & $3,9 \pm 0,7$ & 0,31 \\
SÓDIO (mmol/L) & $140,2 \pm 1,4$ & $133,1 \pm 5,6$ & 0,001 \\
ALBUMINA (g/dL) & $4,2 \pm 0,2$ & $3,4 \pm 0,6$ & 0,001 \\
TGO (U/L) & $33,0 \pm 7,5$ & $28,2 \pm 21,8$ & 0,003 \\
TGP (U/L) & $33,3 \pm 5,0$ & $30,2 \pm 24,0$ & 0,08 \\
GAMA GT (U/L) & $35,8 \pm 4,5$ & $130,5 \pm 122,6$ & 0,001 \\
TSH ( $\boldsymbol{\mu U} / \mathbf{m l})$ & $2,28 \pm 1,0$ & $1,41 \pm 0,4$ & 0,001 \\
T4L (ng/dL) & $1,10 \pm 0,15$ & $0,93 \pm 0,2$ & 0,002 \\
COLESTEROL TOTAL (mg/dL) & $163,3 \pm 18,5$ & $140 \pm 60$ & 0,1 \\
HDL (mg/dL) & $44,2 \pm 7,9$ & $30,6 \pm 12,7$ & 0,003 \\
LDL (mg/dL) & $123,6 \pm 13,6$ & $76,9 \pm 33,5$ & 0,001 \\
TRIGLICERÍDEOS (mg/dL) & $133,0 \pm 23,2$ & $202,9 \pm 194,2$ & 0,08 \\
\hline
\end{tabular}

Legenda: $\mathrm{mm}^{3}$ : milímetro cúbico; g: grama; dl: decilitro; mg: miligrama; mmol: milimol; U: unidades; $\mathrm{L}$ : litro; $\mu \mathrm{U}$ : microunidades; ng: nanogramas.

Fonte: Dados da pesquisa

Quando avaliados os pacientes portadores da síndrome da imunodeficiência adquirida, agrupados em eunatremia (GI) e em hiponatremia (GII), não foram encontradas diferenças estatísticas nos dados demográficos, bem como para o tempo de diagnóstico e de tratamento com antirretrovirais (Tabela 2).

Tabela 2 - Dados demográficos dos pacientes portadores da síndrome da imunodeficiência adquirida com eunatremia (GI) e dos pacientes com hiponatremia (GII) internados na enfermaria de doenças infecciosas de um hospital público terciário de referência para o tratamento de pacientes portadores de HIV/SIDA, localizado em Aracaju - Sergipe, no período de agosto a novembro de 2016

\begin{tabular}{cccc}
\hline & GI $(\mathbf{n}=\mathbf{8})$ & GII $(\mathbf{n}=\mathbf{1 1})$ & $\mathbf{p}$ \\
\hline SEXO (M:F) & $3: 5$ & $8: 3$ & 0,2 \\
IDADE (Anos) & $39,5 \pm 11,3$ & $38,1 \pm 10,8$ & 0,6 \\
PESO (kg) & $62,0 \pm 14,8$ & $60,2 \pm 12,5$ & 0,6 \\
ALTURA (m) & $1,58 \pm 0,09$ & $1,64 \pm 0,09$ & 0,6 \\
IMC (kg/m2) & $24,8 \pm 0,6$ & $22,4 \pm 0,4$ & 0,2 \\
TEMPO DE DIAGNÓSTICO (Meses) & $63,3 \pm 52,5$ & $45,2 \pm 34,5$ & 0,37 \\
\hline
\end{tabular}




\begin{tabular}{cccc}
\hline & GI $(\mathbf{n}=\mathbf{8})$ & GII $(\mathbf{n}=\mathbf{1 1})$ & p \\
\hline TEMPO DE TRATAMENTO (Meses) & $58,6 \pm 56,5$ & $44,9 \pm 34,9$ & 0,51 \\
\hline
\end{tabular}

Legenda: M: masculino; F: feminino; kg: quilograma; m: metro

Fonte: Dados da pesquisa

Nos resultados referentes aos parâmetros bioquímicos avaliados em ambos os grupos foi observada diferença estatística nos níveis de gama-GT e sódio (Tabela 3).

Tabela 3 - Parâmetros bioquímicos avaliados dos pacientes portadores da síndrome da imunodeficiência adquirida com eunatremia (GI) e com hiponatremia (GII) internados na enfermaria de doenças infecciosas de um hospital público terciário de referência para o tratamento de pacientes portadores de HIV/SIDA, localizado em Aracaju - Sergipe, no período de agosto a novembro de 2016

\begin{tabular}{|c|c|c|c|}
\hline & GI $(n=8)$ & GII $(n=11)$ & $\mathbf{p}$ \\
\hline HEMACIA (milhões/mm3) & $4,5 \pm 0,5$ & $3,54 \pm 0,6$ & 0,45 \\
\hline HEMOGLOBINA (g/dL) & $13,2 \pm 0,6$ & $10,3 \pm 2,6$ & 0.80 \\
\hline HEMATÓCRITO (\%) & $40,3 \pm 1,9$ & $31,4 \pm 7,1$ & 0,70 \\
\hline LEUCOCITOS (mm3) & $8393 \pm 2733$ & $8877 \pm 5039$ & 0,45 \\
\hline PLAQUETAS (mm3) & $308634 \pm 81351$ & $296818 \pm 240425$ & 0,25 \\
\hline UREIA (mg/dL) & $37,6 \pm 2,7$ & $34,3 \pm 14,8$ & 0,5 \\
\hline CREATININA (mg/dL) & $0,75 \pm 0,3$ & $0,92 \pm 0,5$ & 0,36 \\
\hline POTÁSSIO (mmol/L) & $3,6 \pm 0,4$ & $4,0 \pm 0,8$ & 0,14 \\
\hline SÓDIO (mmol/L) & $136,9 \pm 1,5$ & $130,4 \pm 4,9$ & 0,007 \\
\hline ALBUMINA (g/dL) & $3,4 \pm 0,7$ & $3,7 \pm 0,6$ & 0,96 \\
\hline $\mathrm{TGO}(\mathrm{U} / \mathrm{L})$ & $20,5 \pm 6,4$ & $33,7 \pm 27,3$ & 0,19 \\
\hline $\mathrm{TGP}(\mathrm{U} / \mathrm{L})$ & $23,9 \pm 17,8$ & $34,8 \pm 27,4$ & 0,33 \\
\hline GAMA-GT (U/L) & $82,9 \pm 94,9$ & $165,1 \pm 13,2$ & 0,03 \\
\hline $\mathrm{TSH}(\mu \mathrm{U} / \mathrm{ml})$ & $1,48 \pm 0,3$ & $1,36 \pm 0,5$ & 0,67 \\
\hline T4L (ng/dL) & $0,91 \pm 0,1$ & $0,94 \pm 0,2$ & 0,60 \\
\hline COLESTEROL TOTAL (mg/dL) & $148,6 \pm 64,8$ & $133,7 \pm 58,2$ & 0,22 \\
\hline $\mathrm{HDL}(\mathrm{mg} / \mathrm{dL})$ & $32,5 \pm 13,7$ & $29,2 \pm 12,5$ & 0,58 \\
\hline $\mathrm{LDL}(\mathrm{mg} / \mathrm{dL})$ & $73,4 \pm 32,5$ & $79,7 \pm 35,6$ & 0,70 \\
\hline
\end{tabular}




\begin{tabular}{cccc}
\hline & GI $(\mathbf{n}=\mathbf{8})$ & GII $(\mathbf{n}=\mathbf{1 1})$ & p \\
\hline TRIGLICERIDEOS $(\mathrm{mg} / \mathrm{dL})$ & $224,3 \pm 164,9$ & $196,7 \pm 212,8$ & 0,76 \\
\hline
\end{tabular}

mm³: milímetro cúbico; g: grama; dl: decilitro; mg: miligrama; mmol: milimol; U: unidades; L: litro; $\mu \mathrm{U}$ : microunidades; ng: nanogramas

Fonte: Dados da pesquisa

No decorrer do estudo houve óbito de dois pacientes do grupo doença (GS) com hiponatremia (GII).

\section{DISCUSSÃO}

A hiponatremia, definida como uma concentração de sódio maior que $136 \mathrm{mEq} / \mathrm{L}$, representa o distúrbio hidroeletrolítico mais comum em pacientes hospitalizados (DOUGLAS, 2006). Nos portadores de HIV/SIDA, a ocorrência desse distúrbio torna-se ainda mais relevante; visto que, além de compartilhar os mesmos fatores de risco presentes na população em geral, os indivíduos com HIV/ SIDA apresentam causas específicas para seu desenvolvimento, como uso de drogas, síndrome da secreção inapropriada do hormônio antidiurético (SIADH), infecções oportunistas e resistência a glicocorticoides (MENON et al., 2015). Desta forma, pode-se dizer que doentes soropositivos representam uma população de risco para o desenvolvimento de hiponatremia, visto que os níveis séricos de sódio nesses pacientes foram estatisticamente menores quando comparados aos do grupo controle (GC).

No presente estudo, 57,9\% dos pacientes do GS apresentaram hiponatremia no momento da admissão, o que está dentro da faixa relatada na literatura (20-80\%) (UPADHYAY et al., 2009). Essa ampla variação pode ser justificada pelasdiferenças metodológicas e amostrais entre os estudos.

Além dos níveis séricos de sódio reduzidos, os pacientes do GS apresentaram, valores significativamente menores de peso e IMC, em relação ao GC. Essa diferença pode ser explicada pelo estado consumptivo desses indivíduos no momento da internação, em que apresentavam graus variados de desnutrição. Tal situação pode ser ratificada pela maior incidência de hipoalbuminemia (importante marcador bioquímico do estado nutricional) nesse grupo de pacientes.

Ao comparar os parâmetros bioquímicos entre os diferentes grupos verificou-se uma maior prevalência de anemia no GS. A anemia nesses doentes é multifatorial, e pode estar relacionada a efeitos indiretos da infecção pelo HIV, a exemplo de infecções oportunistas, carências nutricionais ou uso de medicações como zidovudina (AZT), ganciclovir e sulfametaxazol/trimetropin (BELLOSO; GLASSOCK, 2016).

Ao agrupar os pacientes com síndrome da imunodeficiência adquirida em dois subgrupos, os hiponatrêmicos e os eunatrêmicos, não foi encontrada diferença estatística referente aos dados demográficos de sexo ou idade, assim como tempo de diagnóstico ou de terapia antirretroviral (TARV). Entretanto, os pacientes HIV com hiponatremia (GII) apresentaram, significativamente, níveis mais elevados de gama-GT quando comparados àqueles com níveis séricos de sódio normais (GI).

Individualmente, a hiponatremia, além de ser o distúrbio eletrolítico mais encontrado na prática clínica, classicamente é utilizada como marcador prognóstico e de morbimortalidade (WALD et al., 
2010; FUNK et al., 2010). Na população HIV/SIDA essa associação ainda é pouco compreendida. No decorrer deste estudo, apenas dois pacientes do GS foram a óbito, o que limitou o reconhecimento da real influência da hiponatremia, como fator de risco indepedente para morte nesses pacientes. Entretanto, o estudo de Tang e colaboradores (1993), demonstrou que doentes soropositivos com hiponatremia tiveram maior tempo de internação e maior mortalidade que os pacientes eunatrêmicos.

Houve, ainda, uma correlação negativa entre hiponatremia e carga viral, o que está em desacordo com os achados de Xu e colaboradores (2014).

Outro dado relatado na literatura é que a hiponatremia pode estar associada a outros distúbrios eletrolíticos como a redução sérica do postássio (LIAMIS et al., 2007). Entretanto, não foi observada uma associação positiva entre hiponatremia e hipocalemia nessa pesquisa.

A alta frequência de indivíduos hiponatrêmicos portadores de HIV/SIDA demonstra que este distúrbio deve ser rotineiramente pesquisado nessa população com o objetivo de diagnosticar e tratar precocemente essa comorbidade, visto que o atraso no seu reconhecimento pode influenciar, de forma negativa, no prognóstico desses doentes.

O presente estudo teve como limitações o reduzido número de participantes, o não estadiamento dos pacientes HIV/SIDA de acordo com a Organização Mundial de Saúde (OMS), ausência da mensuração laboratorial do hormônio antidiurético (ADH), bem como tempo de permanência hospitalar e seguimento dos níveis séricos de sódio durante a hospitalização.

\section{CONCLUSÃO}

Diante da escassez de trabalhos sobre a relação da hiponatremia com o seu impacto na morbimortalidade de pacientes com Vírus da Imunodeficiência Humana / Síndrome da Imunodeficiência Adquirida é necessário que haja novos estudos a fim de melhor compreender os diferentes mecanismos em que a hiponatremia influencia na condição clínica desses pacientes a curto e longo prazo.

Nosso estudo chama atenção, por parte da equipe de saúde, que esta situação clínica é prevalente e merece ser reconhecida como importante fator de comorbidades.

\section{REFERÊNCIAS}

DOUGLAS, I. Hyponatremia: why it matters, how it presents, how we can manage it. Clev Clin J Med, v. 73, n. 3, p. S4, 2006.

FOCACCIA, R.; VERONESI, R. Tratado de infectologia. 3. ed. São Paulo: Atheneu, 2007.

FUNK, G. C. et al. Incidence and prognosis of dysnatremias present on ICU admission. Intens Care Med, v. 36, n. 2, p. 304-311, 2010. 
GINĖS, P.; GUEVARA, M. Hyponatremia in cirrhosis: pathogenesis, clinical significance, and management. Hepatology, v. 48, n. 3, p. 1002-1010, 2008.

GOLDMAN L.; AUSIELLO D. Cecil Medicina. 23. ed. Rio de Janeiro: Elsevier, 2009.

MADARIAGA, H. et al. A rare mechanism of hyponatremia in HIV disease. Am J Case Rep, v. 16, p. $707,2015$.

MALHEIROS, A. J. S. et al. Aumento na taxa de detecção do vírus HIV entre jovens de 15 a 24 anos na região Centro-oeste de 2006 a 2017. Programa de Iniciação Científica-PIC/UniCEUB-Relatórios de Pesquisa, 2019.

MELO, M.C. Epidemiologia da infecção por HIV/AIDS: tendências e sobrevida de pacientes. 2020. 172f. Tese (Doutorado em Saúde Coletiva) - Universidade Estadual de Campinas, Campinas. 2020.

MENON, M.C. et al. A. The management of hyponatremia in HIV disease. J Nephrol, v. 26, n. 1, p. 61-72, 2013.

TANG, W. W. et al. Hyponatremia in hospitalized patients with the acquired immunodeficiency syndrome (AIDS) and the AIDS-related complex. Am J Med, v. 94, n. 2, p. 169-174, 1993.

UPADHYAY, A. et al. Epidemiology of hyponatremia. Semin Nephrol, v. 29, n. 3, p. 227-238, 2009.

WALD, R. et al. Impact of hospital-associated hyponatremia on selected outcomes. Arch Intern Med, v. 170, n. 3, p. 294-302, 2010.

$\mathrm{XU}$, L. et al. Moderate/severe hyponatremia increases the risk of death among hospitalized Chinese human immunodeficiency virus/acquired immunodeficiency syndrome patients. PLoS One, v. 9, n. 10, p. e111077, 2014. 
1 Médica - Universidade Tiradentes, Aracaju, SE, Brasil. ORCID: 0000-0001-8493-9864.

Email: isabellearaujoos@gmail.com

2 Graduando em Medicina - Universidade Tiradentes, Aracaju, SE, Brasil. ORCID: 0000-0003-1496-9333.

Email: dsstos1998@gmail.com

3 Doutor em Medicina - Clínica Médica. Departamento de Medicina Interna e Patologia, Centro de Ciências Biológicas e da Saúde, Universidade Federal de Sergipe, Aracaju, SE. Brasil. ORCID: 0000-0002-8464-6188.

Email: frasp@terra.com.br

4 Médico. Universidade Federal de Sergipe, Aracaju, SE, Brasil. ORCID: 0000-0003-4461-4891.

Email: Iuan lipe@hotmail.com

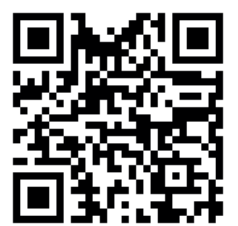

A autenticidade desse artigo pode ser conferida no site https://periodicos. set.edu.br

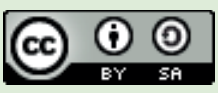

Este artigo é licenciado na modalidade acesso abertosob a Atribuição-Compartilhalgual CC BY-SA
GRUPO TIRADENTES Inspirando ofuturo

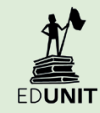

